\title{
BMJ Open Cost-effectiveness of telehealth for patients with raised cardiovascular disease risk: evidence from the Healthlines randomised controlled trial
}

\author{
Padraig Dixon, ${ }^{1}$ Sandra Hollinghurst, ${ }^{1}$ Louisa Edwards, ${ }^{1}$ Clare Thomas, ${ }^{1}$ \\ Daisy Gaunt, ${ }^{2}$ Alexis Foster, ${ }^{3}$ Shirley Large, ${ }^{4}$ Alan A Montgomery, ${ }^{2,5}$ \\ Chris Salisbury ${ }^{1}$
}

To cite: Dixon $P$,

Hollinghurst S, Edwards L, et al. Cost-effectiveness of telehealth for patients with raised cardiovascular disease risk: evidence from the Healthlines randomised controlled trial. BMJ Open 2016;6:e012352.

doi:10.1136/bmjopen-2016012352

- Prepublication history and additional material is available. To view please visit the journal (http://dx.doi.org/ 10.1136/bmjopen-2016012352).

Received 27 April 2016 Revised 2 June 2016 Accepted 29 June 2016

CrossMark

For numbered affiliations see end of article.

Correspondence to

Dr Padraig Dixon;

Padraig.Dixon@bristol.ac.uk

\section{ABSTRACT}

Objectives: To investigate the cost-effectiveness of a telehealth intervention for primary care patients with raised cardiovascular disease (CVD) risk.

Design: A prospective within-trial patient-level economic evaluation conducted alongside a randomised controlled trial.

Setting: Patients recruited through primary care, and intervention delivered via telehealth service.

Participants: Adults with a 10-year CVD risk $\geq 20 \%$, as measured by the QRISK2 algorithm, with at least 1 modifiable risk factor.

Intervention: A series of up to 13 scripted, theory-led telehealth encounters with healthcare advisors, who supported participants to make behaviour change, use online resources, optimise medication and improve adherence. Participants in the control arm received usual care.

\section{Primary and secondary outcome measures:}

Cost-effectiveness measured by net monetary benefit at the end of 12 months of follow-up, calculated from incremental cost and incremental quality-adjusted life years (QALYs). Productivity impacts, participant out-ofpocket expenditure and the clinical outcome were presented in a cost-consequences framework.

Results: 641 participants were randomised-325 to receive the telehealth intervention in addition to usual care and 316 to receive only usual care. $18 \%$ of participants had missing data on either costs, utilities or both. Multiple imputation was used for the base case results. The intervention was associated with incremental mean per-patient National Health Service (NHS) costs of $£ 138(95 \% \mathrm{Cl} 66$ to 211) and an incremental QALY gain of $0.012(95 \% \mathrm{Cl}-0.001$ to $0.026)$. The incremental cost-effectiveness ratio was $£ 10$ 859. Net monetary benefit at a cost-effectiveness threshold of $£ 20000$ per QALY was $£ 116$ (95\% Cl -58 to 291), and the probability that the intervention was cost-effective at this threshold value was 0.77 . Similar results were obtained from a complete case analysis.

Conclusions: There is evidence to suggest that the Healthlines telehealth intervention was likely to be costeffective at a threshold of $£ 20000$ per QALY.

\section{Strengths and limitations of this study}

- We report a within-trial economic evaluation of the one of the largest randomised controlled trials designed to evaluate a telehealth-based complex intervention for the management of cardiovascular disease risk.

- This prospective economic evaluation used detailed patient-level data to contribute to the small body of evidence concerned with the costeffectiveness of telehealth for patients with longterm conditions.

- The intervention was likely to be cost-effective when adopting a UK health system perspective, a conclusion that held whether complete case or imputed data were analysed.

- The recruitment rate to the trial was relatively low, and this may affect the generalisability of these findings.

Trial registration number: ISRCTN27508731; Results. Prospectively registered 05 July 2012.

\section{INTRODUCTION}

Cardiovascular disease (CVD) is the leading cause of death worldwide. ${ }^{1}$ Many of these deaths could be avoided by addressing modifiable behavioural risk factors such as smoking, diet and exercise. ${ }^{1-4}$ Costs associated with CVD are substantial, ${ }^{56}$ and predicted to increase. ${ }^{78}$

There is an urgent need to identify costeffective healthcare interventions that can effect behavioural changes, address the burden of CVD, and efficiently and effectively support patient care. The Healthlines study was a multicentre, parallel two-arm and individually randomised controlled trial (RCT) designed to assess the effectiveness and costeffectiveness of a telehealth intervention for primary care patients. The intervention was 
intended to promote behaviour change, optimisation of medication, improved coordination of care and improved medication adherence in patients with a high risk of developing CVD. Development of the intervention, ${ }^{9}$ the protocol for the trial ${ }^{10}$ and the main results of the trial have been published elsewhere. ${ }^{11}$

Evidence of the effectiveness and cost-effectiveness of telehealth in general, ${ }^{12}$ and for the management of patients with elevated CVD risk, ${ }^{13}$ is mixed. In this paper, we report the results of an economic evaluation conducted alongside the Healthlines RCT. We estimated the cost-effectiveness of the telehealth service from a National Health Service (NHS) perspective for primary care patients with elevated CVD risk. A companion paper $^{14}$ complements the within-trial evaluation presented in this paper with simulation modelling of the cost-effectiveness of this intervention over the remaining lifetime of trial participants.

\section{METHODS}

\section{RCT setting and participants}

Participants aged between 40 and 74 on the date of invitation were recruited from 42 general practices in or near Bristol, Sheffield and Southampton. Participants were individuals with a 10-year risk of a cardiovascular event of $\geq 20 \%$ calculated using the QRISK2 algorithm, ${ }^{15}$ and with at least one of the following modifiable CVD risk factors: (1) systolic blood pressure $\geq 140 \mathrm{~mm} \mathrm{Hg}$, (2) body mass index (BMI) $\geq 30 \mathrm{~kg} / \mathrm{m}^{2}$ and/or (3) a current smoker.

Individuals were excluded from the trial if they had a confirmed diagnosis of CVD (defined as history of heart attack, angina, heart failure, stroke or transient ischaemic attack), currently or planning to be pregnant, or unable to communicate verbally in English. In total, 641 participants were randomised on a 1:1 basis to receive either the telehealth intervention in addition to usual care or usual care alone for up to 12 months.

An NHS perspective was adopted for the costeffectiveness analysis, which compared NHS costs with quality-adjusted life years (QALYs) over the 12 months of trial follow-up. Self-reported data on personal expenditure and productivity impacts were also collected and are presented as part of a cost-consequences analysis.

\section{Intervention}

A structured programme of work (C Salisbury, A O'Cathain, C Thomas, et al. Telehealth for patients with long-term health conditions: development and evaluation of the Healthlines Service. NIHR Journals LibraryProgramme Grants for Applied Research, Under review) was used to develop the de novo, theory-driven, web and phone-based telehealth service received by participants in the intervention arm. The core feature of the Healthlines Service consisted of scripted telephone support and responsive advice delivered by NHS Direct Health Information Advisors (HIAs). The scripts used in the telephone encounters, based on a successful US intervention, ${ }^{16}{ }^{17}$ focused on goal setting, stimulus control and problem solving to address modifiable risk factors for CVD. These encounters were responsive to need, and participants could, for example, request calls with supervisors, and could ask to be directed to sources of information relevant to the management of their condition. Participants were eligible to receive up to 13 scheduled telephone encounters delivered approximately every 4 weeks over the course of the 12 months of trial follow-up. The intervention was in addition to usual care.

Participants with systolic blood pressure $\geq 140 \mathrm{~mm} \mathrm{Hg}$ and without atrial fibrillation were offered a home blood pressure monitor. Blood pressure was reviewed during each encounter with the Healthlines advisor and targets were set based on guidelines issued by the National Institute for Health and Care Excellence (NICE). ${ }^{18}$ General practitioners were advised if patients were not adherent to medication. Participants in the intervention arm were provided with access to an online web portal containing summaries of progress (such as graphs of blood pressure against target), and access to online resources relevant to the management of their condition.

Participants in the control arm received unmodified usual care.

\section{Measurement and valuation of outcomes}

The primary outcome of the RCT was the proportion of participants responding to treatment, defined as a binary outcome reflecting maintenance or reduction of 10-year CVD risk estimated using the QRISK2 algorithm at 12 months after randomisation. QRISK2 scores at 12 months were calculated by updating age and modifiable risk factors, with all other variables held constant.

Participants responded to questions concerning health-related quality of life at baseline and at 6 and 12 months postrandomisation using the EQ-5D-5L measure. ${ }^{19}$ This generic instrument measures five dimensions (mobility, self-care, usual activities, pain and discomfort) of health-related quality of life, and uses five categories to characterise health states associated with these dimensions: no problems, slight problems, moderate problems, severe problems and extreme problems. The EQ-5D-5L UK valuations were not available to us at the time of trial analysis, and hence the EQ-5D-5L responses were 'cross-walked' to the three-level version of the instrument (EQ-5D-3L) and valued using the Euroqol value set for the UK. ${ }^{20}$

\section{Measurement and valuation of resource use}

Relevant primary care consultations and information on prescriptions related to CVD risk (lipid regulating, antihypertensives, antiplatelet, obesity treatments and nicotine dependence medication) were collected (with consent) from medical records. Primary care consultations were costed using Curtis. ${ }^{21}$ Prescribed medications were costed using the Prescription Cost Analysis England (PCAE) database, ${ }^{22}$ and checked against the British National Formulary. $^{23}$ 
Questionnaires issued to participants at 6 and 12 months were used to collect information on healthcare use associated with CVD risk not available from primary care records. Examples of these resources include hospital and ambulance use, district nurse consultations, and use of NHS walk-in centres. Since we imputed costs rather than resource use per se for our base case analysis, we present resource use for available and complete cases in the online supplementary material rather than in the main text.

The sources used to value these resources uses were primarily Curtis, ${ }^{21}$ NHS National Reference Costs for $2012 / 2013^{24}$ and other sources as described in the online supplementary tables A1 and A2. Participants also reported use of private health services (eg, private nutritionists), and out-of-pocket expenditure (eg, selfhelp books) associated with CVD risk. Participants reported time absent from work due to CVD risk, which was valued, where appropriate, at the national median gross hourly wage for 2013 of $£ 11.59 .{ }^{25}$

The estimated cost of the intervention was based on the number and length of telephone calls, the number of failed attempts made by HIAs to contact participants, blood pressure monitors and the costs associated with establishing the service, such as training costs. Unit costs for these resources are described in the online supplementary table A3.

HIAs worked 40 hours per week, and were remunerated at band 4 of the NHS 'Agenda for Change' pay scale. Salaries and associated costs (such as overhead) were based on Curtis. ${ }^{21}$ The cost per hour of participant contact time was estimated based on a ratio of contact to non-contact time calculated from task-scheduling diaries. Initial and ongoing training of HIAs was provided by a nurse-grade trainer; the training was assumed to last for 3 years and costs were amortised on that basis. This assumes that training of this type would not be redundant after 1 year; instead, it assumes that the training would be effective for 3 years, after which additional training might become necessary.

Costs are reported in 2012/2013 pounds sterling.

\section{Analysis of data}

Summary statistics were estimated for all variables used in the economic analysis, and any missingness was identified. All cost-effectiveness analysis was conducted on an 'intention-to-treat' basis. Neither costs nor benefits were discounted given the follow-up period of the trial.

A very small proportion of patients $(<0.5 \%)$ had missing data on primary care consultations. This was primarily due to patients having moved during the study to different practices. Mean values were imputed for these patients. Complete cases refer to cases constructed after initial data cleaning and mean imputation of the small amount of missing primary care consultation data. These complete cases had no other missing cost or EQ-5D-5L data needed to undertake inferential costeffectiveness analysis from the perspective of the NHS.
Approximately $82 \%$ of participants were categorised as complete cases under this definition.

Multiple imputation was used for all other missing data. Aggregate costs at the level of primary care, medication, other NHS costs and responses to the EQ-5D-5L at each time point were imputed using the-icecommand $^{2627}$ in Stata V.13.1, which implements multiple imputation by chained equations.

The imputation model included demographic, cost and clinical outcome variables at baseline and follow-up, stratified by trial arm. Predictive mean matching ${ }^{27}$ was applied to account for non-Gaussian distributions of some included variables, and passive imputation was used for those categorical variables that were functions of other included variables.

The number of imputations $(n=60)$ was selected to be greater than the proportion of missing data $(18 \%) .{ }^{27} \mathrm{We}$ followed the approach set out in Faria $e t a l^{28}$ in order to implement 'Rubin's rules', so that variation within and between the set of 60 imputed data sets was reflected in the analysis of cost-effectiveness.

Health-related quality of life utilities were measured at baseline, and 6 and 12 months using responses to EQ-5D-5L questionnaires. QALYs were calculated from these responses using the 'area under the curve' method, ${ }^{29}$ and were adjusted for baseline differences in EQ-5D-5L scores. ${ }^{30}$

The cost-effectiveness analysis used seemingly unrelated regression (SUR), for which we rely on the near normality of differences in sample means and differences in sample variances in large samples. ${ }^{31} 32$ We implemented SUR using the-sureg-command in Stata. We regressed costs and QALYs on a binary variable indicating allocation to trial arm. For the QALY equation, we also included controlled for baseline imbalances in utility. ${ }^{30}$ No other covariates were included in these regressions.

Incremental cost-effectiveness ratios (ICERs), costeffectiveness acceptability curves (CEACs), net monetary benefit estimates ${ }^{33}$ and CIs around net monetary benefit point estimates were calculated parametrically from regression output. Net monetary benefit was estimated at the NHS threshold values suggested by NICE of

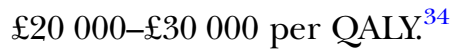

The cost-consequences analysis used all available cases, which were identified on a variable-by-variable basis. These were defined as having complete data across all relevant time points for any individual variable.

Stata V.13.1 software (Statacorp: College Station, Texas, USA) was used in all analyses.

\section{Sensitivity analysis}

We carried out sensitivity analyses in two areas of uncertainty to test the robustness of our results. First, complete case analysis was conducted as a check on the base case imputed cost-effectiveness analysis. Second, the base case (imputed) results were assessed for their sensitivity to self-reported use of secondary care in order to 
assess the effect of rare but expensive events and to address potential recall bias or misclassification of resource use.

\section{RESULTS}

The trial recruited a total of 641 participants: 325 were randomised to receive the intervention and 316 received usual care in the control arm. The mean age of participants in the trial was 67.2 years, of whom $80 \%$ were male, and $99 \%$ of all participants were of white ethnicity. Mean QRISK2 score at baseline in the usual care arm was 30.8 (SD 9.5), and 31.1 (SD 10.2) in the intervention arm.

Compliance with the intervention was reasonably high; $8 \%$ of participants received two or fewer encounters with the HIAs (including the initial introductory encounter), $60 \%$ received 3-11 encounters and 32\% received 12-13 encounters. The median number of encounters received was 10 (IQR 8-12). Two participants had missing data on the number of encounters received.

\section{Outcomes}

There was an imbalance in the baseline imputed EQ-5D-5L scores between the two groups $(0.77$ (SE 0.01 ) in the control group vs 0.80 (SE 0.1) in the intervention group). Once this was adjusted for the size of the QALY gain was 0.012 in the imputed data set used for the base case analysis (table 1). Response to treatment, measured as the maintenance or reduction of QRISK2 scores, was modestly higher in the intervention arm (adjusted OR 1.3, 95\% CI 1.0 to 1.9 ).

\section{Resource use and cost}

The mean cost per participant of providing the intervention was estimated to be $£ 129$ (SD £56.33), of which the cost of the encounter calls constituted $85 \%$ of total cost (table 2). NHS costs (primary care consultations, medication costs, use of NHS community services and NHS secondary care) were similar in each arm (table 3). Online supplementary tables A4-A10 provide disaggregated data on resource use and costs for available and complete cases (as defined above).

\section{Cost-consequences}

Table 4 presents a cost-consequences matrix showing costs from different perspectives and a range of outcomes.

\begin{tabular}{|c|c|c|}
\hline Cost and outcomes & $\begin{array}{l}\text { Usual care } \\
\left(n=316^{*}\right) \\
\text { mean (SE) }\end{array}$ & $\begin{array}{l}\text { Intervention } \\
\left(n=325^{*}\right) \\
\text { mean (SE) }\end{array}$ \\
\hline Imputed unadjusted QALYs & $0.774(0.100)$ & $0.810(0.009)$ \\
\hline $\begin{array}{l}\text { Imputed QALYs, adjusted } \\
\text { for baseline imbalance }\end{array}$ & $0.786(0.005)$ & $0.798(0.005)$ \\
\hline
\end{tabular}

Table 2 Mean (SD) intervention cost (£) per participant for all participants and complete cases

\begin{tabular}{|c|c|c|}
\hline $\begin{array}{l}\text { Intervention } \\
\text { elements }\end{array}$ & $\begin{array}{l}\text { All participants } \\
(n=325) \text { mean } £ \\
(S D)\end{array}$ & $\begin{array}{l}\text { Complete cases } \\
(n=262) \text { mean } £ \\
(S D)\end{array}$ \\
\hline Encounter calls & $108.80(49.75)$ & $114.68(46.07)$ \\
\hline Non-scheduled calls & $1.39(2.57)$ & $1.47(2.65)$ \\
\hline All calls & $110.20(50.13)$ & $116.15(46.24)$ \\
\hline $\begin{array}{l}\text { Blood pressure } \\
\text { monitor }\end{array}$ & $18.92(18.78)$ & 18.89 (18.79) \\
\hline $\begin{array}{l}\text { Total cost per } \\
\text { participant }\end{array}$ & $129.12(56.33)$ & 135.04 (53.02) \\
\hline
\end{tabular}

Costs to the NHS were higher in the intervention arm, largely due to the cost of the intervention. The societal value of lost production was slightly higher per patient in the control arm, but not significantly so. The comparison is affected by the low numbers of participants reporting employment at either 6 or 12 months $(25.2 \%)$, and the number of employed participants reporting no impact $(95.1 \%)$ of CVD risk factors on their employment. Participants randomised to usual care reported higher mean per patient private healthcare costs than in the intervention arm in available cases, but lower out-of-pocket expenditure than intervention participants.

These costs were associated with a marginal increase in the proportion of responders (defined in the published trial protocol as the maintenance or reduction of 10-year cardiovascular risk estimated on the basis of the QRISK2 score after 12 months) in the intervention arm, with improvements in EQ-5D-5L utility at the end of 12 months of follow-up, and with a small QALY improvement.

There was a marginal increase in response to treatment (as defined above) in the treatment arm. The intervention was also associated with reductions in blood pressure (mean difference in systolic pressure $-2.7 \mathrm{~mm} \mathrm{Hg} ; 95 \%$ CI -4.7 to -0.6$)$ and in weight $(-1.0 \mathrm{~kg} ; 95 \%$ CI -1.8 to -0.3$)$. Improvements in diet, physical activity, medication to care and satisfaction with treatment for participants randomised to the intervention arm were also observed. The intervention did not improve cholesterol or smoking status.

\section{Cost-effectiveness}

Cost-effectiveness results from an NHS perspective are presented in table 5. The ICER is $£ 10859$ and there is a probability of 0.77 that the intervention is cost-effective at a threshold value of $£ 20000$ per QALY. The probability that the intervention is cost-effective at other values of the cost-effectiveness threshold is shown in figure 1. The between-arm QALY difference of 0.012 corresponds to $\sim 4$ additional days in 'perfect' health over the course of a year for participants randomised to the intervention arm rather than to the control arm. 
Table 3 Imputed NHS costs

\begin{tabular}{lllc}
\hline Cost and outcomes & $\mathbf{N}^{*}$ & $\begin{array}{l}\text { Usual care mean } £ \\
\text { (standard error)† }\end{array}$ & $\begin{array}{c}\text { Intervention mean } £ \\
\text { (standard error)† }\end{array}$ \\
\hline Imputed hospital, ambulance and other mean NHS costs & 641 & $56(19)$ & $65(22)$ \\
Imputed mean drug costs & 641 & $67(8)$ & $67(6)$ \\
Imputed mean primary care costs & 641 & $241(11)$ & $242(9)$ \\
Imputed mean NHS costs, excluding cost of the intervention & 641 & $364(26)$ & $373(26)$ \\
Imputed mean intervention costs & 641 & - & $129(3)$ \\
Imputed mean NHS-related costs, including cost of the & 641 & $364(26)$ & $502(27)$ \\
intervention & & & \\
*This sample size is based on 60 imputed datasets. & & \\
†Standard errors are reported for imputed data, rather standard deviations.
\end{tabular}

Table 4 Cost-consequence matrix

\begin{tabular}{|c|c|c|c|c|c|}
\hline Cost and outcomes & $\begin{array}{l}\text { Usual } \\
\text { care }\end{array}$ & $\mathbf{N}$ & Intervention & $\mathbf{N}$ & Difference $(95 \% \mathrm{Cl})$ \\
\hline \multicolumn{6}{|l|}{ Available data on costs $(£)$} \\
\hline Mean cost of intervention & 0 & 316 & 129 & 325 & - \\
\hline $\begin{array}{l}\text { Mean cost of NHS resources, } \\
\text { excluding intervention costs }\end{array}$ & 361 & 283 & 362 & 285 & $1(-72 \text { to } 76)^{*}$ \\
\hline $\begin{array}{l}\text { Mean cost of NHS resources, } \\
\text { including intervention costs }\end{array}$ & 361 & 283 & 494 & 283 & $132(57 \text { to } 212)^{*}$ \\
\hline Out-of-pocket expenses & 64 & 298 & 79 & 299 & $15(-20 \text { to } 50)^{*}$ \\
\hline Private healthcare & 110 & 298 & 59 & 299 & $-50(-141 \text { to } 1)^{\star}$ \\
\hline Mean societal value per patient of lost production & 76 & 298 & 52 & 299 & $-24(-133 \text { to } 54)^{*}$ \\
\hline \multicolumn{6}{|l|}{ Consequencest } \\
\hline $\begin{array}{l}\text { QRISK2 response to treatment (proportion of } \\
\text { responders) }\end{array}$ & $43 \%$ & 291 & $50 \%$ & 295 & Adjusted OR 1.3 (1.0 to 1.9 ) \\
\hline EQ-5D-5L at 12 months, unadjusted for baseline $\ddagger$ & 0.776 & 297 & 0.812 & 295 & $0.037(0.007 \text { to } 0.070)^{*}$ \\
\hline QALYs, adjusted for baseline $\neq$ & 0.788 & 279 & 0.799 & 275 & $0.01(-0.014 \text { to } 0.040)^{*}$ \\
\hline
\end{tabular}

${ }^{*} \mathrm{Cl}$ calculated as accelerated and bias corrected interval from 1000 bootstrap replicates to account for skewed distributions.

†All consequences measured at 12 months, or over a period of 12 months.

$\ddagger$ Based on available data.

NHS, National Health Service; QALYs, quality-adjusted life years.

Table 5 Cost-effectiveness of the intervention from an NHS perspective

\begin{tabular}{|c|c|c|c|}
\hline Cost of services & Usual care mean & Intervention mean & Incremental difference $(95 \% \mathrm{Cl})$ \\
\hline \multicolumn{4}{|l|}{ Costs and QALYs } \\
\hline Total NHS costs & $£ 364$ & $£ 502$ & $£ 138(66$ to 211$)$ \\
\hline Adjusted QALYs & 0.786 & 0.798 & $0.012(-0.001$ to 0.026$)$ \\
\hline \multicolumn{4}{|c|}{ Cost-effectiveness statistics } \\
\hline \multicolumn{4}{|c|}{ ICER: $£ 10859$} \\
\hline \multicolumn{4}{|c|}{ Probability that intervention cost-effective at CE threshold of $£ 20000: 0.77$} \\
\hline \multicolumn{4}{|c|}{ Probability that intervention cost-effective at CE threshold of $£ 30000: 0.87$} \\
\hline \multicolumn{4}{|c|}{ NMB at threshold of $£ 20000(95 \% \mathrm{Cl}): £ 116$ (105 to 128$)$} \\
\hline
\end{tabular}

\section{Sensitivity analyses}

The base case results are insensitive to the exclusion of secondary NHS costs. The effect of removing these costs is to slightly narrow cost differences between arms (relative to the base case) so that the ICER reduces to
$£ 10003$, and hence these costs do not have a material impact on the likely cost-effectiveness of the intervention.

Table 6 presents the results of a complete case analysis of cost-effectiveness from an NHS perspective. The CEAC associated with these results is presented as online 


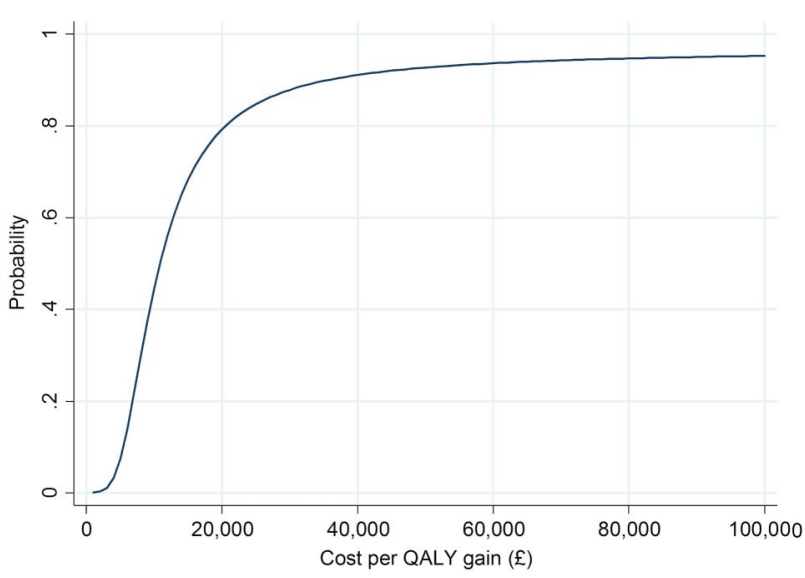

Figure 1 Cost-effectiveness acceptability curve from an NHS perspective for imputed model. NHS, National Health Service; QALY, quality-adjusted life year.

supplementary figure A1. The complete case results are similar to those of the imputed base case results.

\section{DISCUSSION}

We conducted a within-trial evaluation of costeffectiveness of a de novo telehealth intervention in patients with elevated CVD risk. Trial participants in the control arm received usual care. Participants were followed up for 12 months. Resource use data were collected at 6 and 12 months, and information on health-related quality of life was measured using responses to the EQ-5D-5L questionnaire. Using a health system perspective, we compared healthcare costs to QALYs in the base case cost-effectiveness analysis.

The between-arm difference in QALYs is modest. This small improvement in QALYs in the intervention arm may reflect the indirect impact of engaging with and being supported by the HIAs. For example, intervention participants reported greater medication adherence, better access to support and satisfaction with treatment than did control participants (C Salisbury, A O'Cathain, C Thomas, et al. Under review). Intervention participants reported small improvements in blood pressure and BMI, but not with respect to smoking or cholesterol
(C Salisbury, A O'Cathain, C Thomas, et al. Under review). The balance of these impacts-on the sense of being supported, greater satisfaction with treatment and some improvements in 'hard' health indicators such as weight-may have contributed to EQ-5D-5L responses that favoured the intervention.

\section{Strengths}

The Healthlines trial was one of the largest RCTs designed to evaluate a telehealth-based complex intervention for the management of CVD risk. The intervention was based on a programme of work (C Salisbury, A O'Cathain, C Thomas, et al. Under review) intended to establish the acceptability of telehealth to patients with chronic conditions, and to support the development of an evidence-based, responsive telehealth service for evaluation in the context of a pragmatic trial design. This economic evaluation contributes to the small body of evidence concerned with the cost-effectiveness of telehealth for patients with long-term conditions.

The economic evaluation was conducted alongside the trial, and prospectively collected detailed patientlevel data. The analysis was conducted in line with best practice guidance. ${ }^{35}$ The amount of missing data in the cost and quality of life variables necessary to conduct inferential cost-effectiveness analysis was similar in each arm. Imputed and complete case cost-effectiveness results were similar.

Adherence to the intervention was relatively strong, with a median of 10 out of 13 scheduled encounters received. This suggests that patients randomised to the intervention were willing to engage with the Healthlines Service.

\section{Limitations}

The economic evaluation did not seek to measure the scalability of the intervention to larger (eg, national) patient groups, although the intervention was specifically designed to be easily scaled-up. It is conceivable that scale economies (eg, training more HIAs at the same cost) and other savings (eg, through very large bulk purchases of blood pressure monitors) could be

\begin{tabular}{|c|c|c|c|}
\hline Cost of services & Usual care $(n=266)$ mean & Intervention $(n=262)$ mean & $\begin{array}{l}\text { Incremental difference } \\
(95 \% \mathrm{Cl})\end{array}$ \\
\hline \multicolumn{4}{|l|}{ Costs and QALYs } \\
\hline Total NHS costs-complete case & $£ 367$ & $£ 490$ & $£ 124$ (42 to 206$)$ \\
\hline QALYs-complete case & 0.788 & 0.800 & $0.011(-0.001$ to 0.025$)$ \\
\hline \multicolumn{4}{|l|}{ Cost-effectiveness statistics } \\
\hline \multicolumn{4}{|c|}{ ICER: $£ 10366$} \\
\hline \multicolumn{4}{|c|}{ Probability that intervention cost-effective at CE threshold of $£ 20000: 0.79$} \\
\hline \multicolumn{4}{|c|}{ Probability that intervention cost-effective at CE threshold of $£ 30000: 0.87$} \\
\hline \multicolumn{4}{|c|}{ NMB at threshold of $20000(95 \% \mathrm{Cl}): £ 115(103$ to 127$)$} \\
\hline
\end{tabular}


realised to reduce overall intervention cost. There was no evidence from the trial that substantial efficiencies could have been secured but were left unexploited. A related limitation is that the recruitment rate of the trial was relatively low. ${ }^{11}$ This will affect the generalisability of the findings, although it is unclear whether low reluctance is due to lack of interest in telehealth or unwillingness to participate in research.

The 12-month follow-up period of the trial means that questions concerning long-term outcomes cannot be answered definitively, and nor can the issue of whether time-limited (eg, for 12 months) or ongoing (eg, until QRISK2 score was reduced to some target level) telehealth support would be most cost-effective. The intervention may affect the health of participants beyond the end of follow-up for at least two reasons: any improvements in habits and self-management are maintained, and the reduced QRISK2 scores in the intervention during trial follow-up will (slightly) reduce the likelihood of future CVD events occurring. A companion paper ${ }^{14}$ describes the results of a simulation intended to assess the cost-effectiveness of the intervention from a lifetime perspective.

\section{Other literature}

Incremental costs per patient in the intervention arm were £138. There was a small QALY difference between arms of 0.012 . We note that Bergmo's ${ }^{36}$ review of QALY gains in telehealth interventions found that 17 included studies reported small, positive effects on quality of life. This is consistent with the results reported here. Overall, the intervention is likely to be cost-effective at conventional NHS costeffectiveness thresholds.

Comparison of the findings of this economic evaluation with other literature is complicated by differences between studies in technologies assessed, the types of condition analysed, study design and patient population. Evidence for the cost-effectiveness of telehealth in general is mixed, ${ }^{36-39}$ and has been described as being of low quality in some cases. ${ }^{36} 40-42$

A notable example of cost-effectiveness analysis within an English NHS context of telehealth for longterm conditions was evaluation of the "Whole Systems Demonstrator' (WSD) project. ${ }^{43} 44$ Patients with a long-term condition (heart failure, chronic obstructive pulmonary disease or diabetes) received either telehealth $(n=845)$ or usual care $(n=728)$ for 12 months following cluster randomisation of practices in three study sites.

The WSD intervention comprised the use of a broad class of telehealth and telemonitoring equipment, which differed by study site. Participants were asked to take readings using this equipment at the same time each day up to 5 days per week. Specialist nurses monitored and responded to information from patients.

The adjusted estimated QALY gain after 12 months in the WSD study was 0.012 in favour of the intervention, the same mean difference reported here for Healthlines, despite the differences in technology, patient characteristics and study design. NHS and social care costs were substantially higher than in Healthlines, with WSD intervention arm costs $£ 1110$ higher than in the control arm. The costs of the intervention itself were estimated to be substantially higher than in Healthlines (£1847 average annual cost for those with equipment and reporting costs at 12-month follow-up in WSD, compared with $£ 129$ in Healthlines), although other costs were lower in the intervention arm. The estimated WSD ICER was $£ 92000$.

This high-level comparison between the costeffectiveness results of the Healthlines trial and from the WSD trial indicate the sensitivity of the cost-effectiveness results to the type and costs of technology evaluated. Despite the same mean QALY difference, the WSD evaluation suggested that substantial changes in intervention cost would be necessary before the service could be considered as a cost-effective means of managing patients with long-term conditions. In contrast to the WSD, the Healthlines intervention was designed to support self-management of chronic diseases at low cost, using readily available low-cost technology such as accessible websites, and telephone support from staff without clinical training.

These considerations are relevant to future study design in this area. Continuing evolution in technology and technology cost is likely to influence both the effectiveness and cost-effectiveness of new interventions.

\section{CONCLUSION}

The Healthlines RCT provided weak evidence of a modest effect on 10-year CVD risk of the telehealth intervention on the primary binary outcome of response to treatment. However, the intervention was estimated to be cost-effective (measured as a function of the ratio of incremental costs to incremental QALYs) from an NHS perspective after 12 months of trial follow-up.

\section{Author affiliations}

${ }^{1}$ Centre for Academic Primary Care, School of Social and Community Medicine, University of Bristol, Bristol, UK

${ }^{2}$ Bristol Randomised Trials Collaboration (BRTC), School of Social and Community Medicine, University of Bristol, Bristol, UK

${ }^{3}$ Clinical Trials Research Unit, School of Health and Related Research (ScHARR), University of Sheffield, Sheffield, UK

${ }^{4}$ Public Health England, Hampshire, UK

${ }^{5}$ Nottingham Clinical Trials Unit, Faculty of Medicine \& Health Sciences, University of Nottingham, Nottingham, UK

Twitter Follow Padraig Dixon at @PadraigDixon

Acknowledgements The authors are very grateful to all patients, healthcare professionals, Health Information Advisors and other National Health Service (NHS) Direct staff who contributed time and effort to make the Healthlines trial possible. The authors are also grateful to administrative staff at trial sites for support with participant recruitment, data entry and trial administration. The Healthlines Study was designed and delivered in collaboration with Bristol 
Randomised Trials Collaboration (BRTC), a UKCRC Registered Clinical Trials Unit in receipt of National Institute for Health Research (NIHR) CTU support funding. The authors are also grateful to Roberta Ara for comments on this manuscript.

Contributors PD and SH conducted economic analysis. SH contributed to protocol development and obtained funding. LE and CT acted as trial mangers. LE also undertook participant recruitment and follow-up, data collection and data entry. AF was involved in participant recruitment and follow-up, data collection and data entry. SL contributed to protocol development, obtained funding and provided methodological advice. DG and AAM were involved in statistical advice and analysis, and provided methodological input into economic analysis. AAM contributed to protocol development and obtained funding. CS led protocol development and the funding application for the programme grant, and acted as chief investigator with overall responsibility for conduct of the randomised controlled trial. All authors had a role in interpreting the data. PD wrote the first draft of this article, and all authors contributed to subsequent revisions. All authors approved the final version for submission.

Funding This report summarises independent research funded by the NIHR under its Programme Grant for Applied Research (Grant Reference Number RP-PG-0108-10011)

Disclaimer The views and opinions expressed in this report are those of the authors and do not necessarily reflect those of the NIHR, the NHS or the Department of Health.

Competing interests None declared.

Patient consent Obtained.

Ethics approval The Healthlines trial was approved by the National Research Ethics Service Committee South West-Frenchay (Reference 12/SW/0009).

Provenance and peer review Not commissioned; externally peer reviewed.

Data sharing statement The data are stored on servers at the University of Bristol. The research team will consider requests for sharing of patient leve data. Consent for data sharing was not obtained but the presented data are anonymised and risk of identification is low. Requests should be made to Professor Chris Salisbury.

Open Access This is an Open Access article distributed in accordance with the terms of the Creative Commons Attribution (CC BY 4.0) license, which permits others to distribute, remix, adapt and build upon this work, for commercial use, provided the original work is properly cited. See: http:// creativecommons.org/licenses/by/4.0/

\section{REFERENCES}

1. World Health Organization. Cardiovascular diseases (CVDs). Secondary cardiovascular diseases (CVDs). 2015. http://www.who. int/mediacentre/factsheets/fs317/en/

2. Santulli G. Epidemiology of cardiovascular disease in the 21st century: updated numbers and updated facts. J Cardiovasc Dis Res 2013;1:2.

3. World Health Organization. Global status report on noncommunicable diseases 2014. Siwtzerland: World Health Organization, 2014

4. Gaziano TA, Bitton A, Anand S, et al. Growing epidemic of coronary heart disease in low- and middle-income countries. Curr Probl Cardiol 2010;35:72-115

5. Leal J, Luengo-Fernandez R, Gray A, et al. Economic burden of cardiovascular diseases in the enlarged European Union. Eur Heart J 2006;27:1610-19.

6. Luengo-Fernández R, Leal J, Gray A, et al. Cost of cardiovascular diseases in the United Kingdom. Heart 2006;92:1384-9.

7. Bloom DE, Cafiero ET, Jané-Llopis E, et al. The global economic burden of noncommunicable diseases. Geneva: World Economic Forum, 2011.

8. Heidenreich PA, Trogdon JG, Khavjou OA, et al. Forecasting the future of cardiovascular disease in the United States: a policy statement from the American Heart Association. Circulation 2011;123:933-44

9. Salisbury C, Thomas C, O'Cathain A, et al. TElehealth in $\mathrm{CHronic}$ disease: mixed-methods study to develop the $\mathrm{TECH}$ conceptual model for intervention design and evaluation. BMJ Open 2015;5: e006448

10. Thomas CL, Man MS, O'Cathain A, et al. Effectiveness and cost-effectiveness of a telehealth intervention to support the management of long-term conditions: study protocol for two linked randomized controlled trials. Trials 2014;15:36.

11. Salisbury C, O'Cathain A, Thomas C, et al. Telehealth for patients at high risk of cardiovascular disease: pragmatic randomised controlled trial. BMJ 2016;353:i2647.

12. Wootton R. Twenty years of telemedicine in chronic disease management-an evidence synthesis. J Telemed Telecare 2012;18:211-20.

13. Merriel SW, Andrews V, Salisbury C. Telehealth interventions for primary prevention of cardiovascular disease: a systematic review and meta-analysis. Prev Med 2014;64:88-95.

14. Dixon $\mathrm{P}$, Hollinghurst $\mathrm{S}$, Ara R, et al. Cost-effectiveness modelling of telehealth for patients with raised cardiovascular disease risk: evidence from a cohort simulation conducted alongside the Healthlines randomised controlled trial. BMJ Open 2016; Under review.

15. Hippisley-Cox J, Coupland C, Vinogradova Y, et al. Predicting cardiovascular risk in England and Wales: prospective derivation and validation of QRISK2. BMJ 2008;336:1475-82.

16. Bosworth $\mathrm{HB}$, Olsen MK, McCant F, et al. Hypertension Intervention Nurse Telemedicine Study (HINTS): testing a multifactorial tailored behavioral/educational and a medication management intervention for blood pressure control. Am Heart $J$ 2007;153:918-24.

17. Bosworth HB, Powers BJ, Olsen MK, et al. Home blood pressure management and improved blood pressure control: results from a randomized controlled trial. Arch Intern Med 2011;171: 1173-80.

18. National Institute for Health and Care Excellence. Hypertension: clinical management of primary hypertension in adults. London: National Institute for Health and Care Excellence, 2011.

19. Herdman M, Gudex C, Lloyd A, et al. Development and preliminary testing of the new five-level version of EQ-5D (EQ-5D-5L). Qual Life Res 2011;20:1727-36.

20. Euroqol. Interim scoring for the EQ-5D-5L: Mapping the EQ-5D-5L to EQ-5D-3L value sets. Secondary interim scoring for the EQ-5D-5L: Mapping the EQ-5D-5L to EQ-5D-3L value sets. http:// www.euroqol.org/fileadmin/user upload/Documenten/PDF/ Crosswalk_5L/EQ-5D-5L_Crosswalk_model_and_methodology.pdf

21. Curtis L. Unit costs of health and social care 2013. Canterbury: Personal Social Services Research Unit, 2013.

22. Stevenson LJ, Coody DK, Evans KD, et al. Providing better access to health care: a pediatric nurse practitioner WIC-based clinic for one-stop health care. J Pediatr Health Care 1994;8:168-72.

23. Joint Formulary Committee. British National Formulary (BNF) 66. Pharmaceutical Press, 2013.

24. Department of Health. Reference costs 2012/13. London, 2013.

25. ONS. Annual survey of hours and earnings, 2013 provisional results ONS, 2013.

26. Royston $\mathrm{P}$, White IR. Multiple Imputation by Chained Equations (MICE): implementation in Stata. J Stat Software 2011; 45:1-20.

27. White IR, Royston P, Wood AM. Multiple imputation using chained equations: issues and guidance for practice. Stat Med 2011;30:377-99.

28. Faria R, Gomes M, Epstein D, et al. A guide to handling missing data in cost-effectiveness analysis conducted within randomised controlled trials. Pharmacoeconomics 2014;32:1157-70.

29. Glick H, Doshi J, Sonnad S, et al. Economic evaluation in clinical trials. Oxford: Oxford University Press, 2007.

30. Manca A, Hawkins N, Sculpher MJ. Estimating mean QALYs in trial-based cost-effectiveness analysis: the importance of controlling for baseline utility. Health Econ 2005;14:487-96.

31. Mihaylova B, Briggs A, O'Hagan A, et al. Review of statistica methods for analysing healthcare resources and costs. Health Econ 2011;20:897-916.

32. Nixon RM, Wonderling D, Grieve RD. Non-parametric methods for cost-effectiveness analysis: the central limit theorem and the bootstrap compared. Health Econ 2010;19:316-33.

33. Drummond M, Sculpher M, Claxton K, et al. Methods for the economic evaluation of health care programmes. 4th edn. Oxford: Oxford University Press, 2015

34. NICE. Guide to the methods of technology appraisal. Manchester, 2013.

35. Husereau D, Drummond M, Petrou S, et al. CHEERS Task Force. Consolidated Health Economic Evaluation Reporting Standards (CHEERS) statement. BMJ 2013;346:f1049. 
36. Bergmo TS. Using QALYs in telehealth evaluations: a systematic review of methodology and transparency. BMC Health Serv Res 2014;14:332.

37. Mistry H, Garnvwa H, Oppong R. Critical appraisal of published systematic reviews assessing the cost-effectiveness of telemedicine studies. Telemed J E Health 2014;20:609-18.

38. Bergmo TS. How to measure costs and benefits of eHealth interventions: an overview of methods and frameworks. J Med Internet Res 2015;17:e254.

39. Mistry H. Systematic review of studies of the cost-effectiveness of telemedicine and telecare. Changes in the economic evidence over twenty years. J Telemed Telecare 2012;18:1-6.

40. Bergmo TS. Can economic evaluation in telemedicine be trusted? A systematic review of the literature. Cost Eff Resour Alloc 2009;7:18.
41. Polisena J, Coyle D, Coyle K, et al. Home telehealth for chronic disease management: a systematic review and an analysis of economic evaluations. Int J Technol Assess Health Care 2009;25:339-49.

42. Udsen FW, Hejlesen O, Ehlers LH. A systematic review of the cost and cost-effectiveness of telehealth for patients suffering from chronic obstructive pulmonary disease. $J$ Telemed Telecare 2014;20:212-20.

43. Steventon A, Bardsley M, Billings J, et al. Effect of telehealth on use of secondary care and mortality: findings from the Whole System Demonstrator cluster randomised trial. BMJ 2012;344:e3874.

44. Henderson $C$, Knapp M, Fernández J-L, et al. Cost effectiveness of telehealth for patients with long term conditions (Whole Systems Demonstrator telehealth questionnaire study): nested economic evaluation in a pragmatic, cluster randomised controlled trial. BMJ 2013;346:f1035 